Review

\title{
MiR-27a: A Novel Biomarker and Potential Therapeutic Target in Tumors
}

\author{
Xingwang $\mathrm{Li}^{1 *}$, Min $\mathrm{Xu}^{1^{*}}$, Li Ding${ }^{2}$, Jinhai Tang ${ }^{1,2}{ }^{\circledR}$ \\ 1. School of Clinical Medicine, Xuzhou Medical University, 209 Tongshan Road, Xuzhou 221004, PR China \\ 2. Department of General Surgery, the First Affiliated Hospital of Nanjing Medical University, Nanjing 210029, P.R. China \\ * These authors contributed equally to this work. \\ $\triangle$ Corresponding author: Jin-Hai Tang, MD, PHD, Department of General Surgery, the First Affiliated Hospital of Nanjing Medical University, Nanjing 210029, \\ P.R. China.E-mail addresses: jhtang@njmu.edu.cn \\ (C) Ivyspring International Publisher. This is an open access article distributed under the terms of the Creative Commons Attribution (CC BY-NC) license \\ (https://creativecommons.org/licenses/by-nc/4.0/). See http://ivyspring.com/terms for full terms and conditions.
}

Received: 2018.11.08; Accepted: 2019.05.09; Published: 2019.06.02

\begin{abstract}
MicroRNAs (miRNAs) are endogenous, time sequencing, conserved and small non-coding RNA molecules (19-25 bp long) that regulate gene expression at the post-transcriptional level by binding to the partial sequence homology of the 3'-untranslated region of target messenger $(\mathrm{m}) \mathrm{RNA}$. The miRNA-27 family consists of miR-27a and miR-27b, which are transcribed from different chromosomes and different in nucleotide at the $3^{\prime}$ end. It has been reported that miR-27a was located on chromosome 19 and played a vital role in tumor development. Increasing evidences support a vital role for miR-27a in modulating polymorphisms, tumorigenesis, proliferation, apoptosis, invasion, migration and angiogenesis. Apart from it, miR-27a could affect drug sensitivity, treatment of cancer and patients prognosis. The miR-27a could be an oncogene or a tumor suppressor in several types of cancer, including colon cancer, pancreatic cancer, breast cancer, bladder cancer and hepatocellular carcinoma. In this review, we discuss the role of miR-27a in tumor biology and clinical significance in detail and offer novel insights into molecular targeting therapy for human cancers.
\end{abstract}

Key words: miRNA, miR-27a, cancer

\section{Introduction}

Cancer is a major progressive health threat with high incidence and mortality all around the world. Moreover, cancer can lead to a tremendous burden on society in every country. Therefore we urgently need to search for innovative treatments and biomarkers for cancers. The role of miRNAs in cancer is a rapidly emerging area of investigation. MicroRNAs (miRs), which are evolutionary conserved, time sequencing and small non-coding RNA molecules, consist of approximately 22 nucleotides to regulate gene expression. MiRs inhibit the gene expression post-transcriptionally by binding specifically to the 3'untranslated-region (UTR) of their target. The miRNA-27 family consists of miR-27a and miR-27b, and the former plays a vital role in tumor development. They are transcribed from different chromosomes and are different in nucleotides at the $3^{\prime}$ end. Increasing evidence has confirmed that miR-27a plays a vital role in tumor biology, including polymorphisms, tumorigenesis, proliferation, apoptosis, invasion, migration and angiogenesis. Besides this, miR-27a has vital clinical significance in drug sensitivity, treatment of cancer and patient's prognosis. In recent studies, miR-27a, was significantly dysregulated in various cancers, such as NSCLC, liver cancer and prostate cancer, and acted as an oncogene. Meanwhile, it could function as a tumor suppressor in gastric cancer, bladder cancer, esophageal squamous cell (Table 1). In this review, we will focus on the action of miR-27a in the processes of tumor biology, such as polymorphisms, tumorigenesis, proliferation, apoptosis, invasion, 
migration and angiogenesis, as well as clinical significance including drug sensitivity, treatment of cancer and patients prognosis, work out the network of genes modulating its functions, and predict the potential role of miR-27a in the diagnosis and molecular targeted therapy of human cancers.

Table 1. The dysregulated miR-27a and its targets in various cancers.

\begin{tabular}{|c|c|c|}
\hline Diseases & Targeted gene & References \\
\hline NSCLC & MET, EGFR & Acunzo et al (31) \\
\hline \multirow{2}{*}{$\begin{array}{l}\text { hepatocellular carcinoma } \\
\text { (HCC) }\end{array}$} & FOXO1 & Sun et al(34) \\
\hline & PPARY & Li et al(35) \\
\hline \multirow[t]{3}{*}{ prostate cancer(PCa) } & РHB & Claire E. Fletcher et al(38) \\
\hline & RUNX-1 & Deng et al(13) \\
\hline & COUP-TFII & Lin et al(42) \\
\hline renal cell carcinoma & EGFR & Li et al(16) \\
\hline $\begin{array}{l}\text { esophageal squamous cell } \\
\text { carcinoma }\end{array}$ & FBXW7 & Wu et al (40) \\
\hline \multirow[t]{2}{*}{ colon cancer } & SGPP1,Smad2 & Bao et al(25) \\
\hline & Calreticulin & TColangelo et al(19) \\
\hline cervical cancer & B4GALT3 & Sun et al(23) \\
\hline \multirow[t]{2}{*}{ oral squamous cell carcinoma } & YAP1 & Zeng et al(41) \\
\hline & MCPH1 & Thejaswini Venkatesh et al(33) \\
\hline \multirow[t]{3}{*}{ breast cancer } & SPRY2 & Li et $\mathrm{al}(20)$ \\
\hline & TMEM170B & Li et al(18) \\
\hline & BAK & Zhou et al(70) \\
\hline gastric cancer & $\mathrm{P} 21$ & Zhao et al(26) \\
\hline ovarian tumor & ZBTB10 & Lai et al(58) \\
\hline tumors or ischemic diseases & SEMA6A & Carmen Urbich et al(44) \\
\hline osteosarcoma & CBFA2T3 & Zaidoun Salah1 et al(43) \\
\hline bronchial cancer & Fbxw7 & Wang et al(22) \\
\hline Glioblastoma (GBM) & MXI1 & Xu et al(29) \\
\hline $\begin{array}{l}\text { esophageal squamous cell } \\
\text { carcinoma }\end{array}$ & KRAS & Jiang et al(17) \\
\hline \multirow{2}{*}{$\begin{array}{l}\text { hepatocellular carcinoma } \\
\text { (HCC) }\end{array}$} & P21 & Sun et al(34) \\
\hline & VE-cadherin & Jennifer A. Young et al(55) \\
\hline bladder urothelial carcinoma & SLC7A11 & Ross M Drayton et al(72) \\
\hline
\end{tabular}

\section{MiR-27a influences tumor biology}

\section{Polymorphism of miR-27a}

Single-nucleotide polymorphisms (SNPs) in miRNA genes were a novel class of genetic variations in the human genome. Increasing studies revealed pre-miR-27a (rs895819) or miR-27a gene (rs11671784) may be associated with cancer risk. They demonstrated that the SNPs could not only enhance or reduce the cancer risk but also exert a vital function in chemo-sensitivity. Recent studies reported that the polymorphism in hsa-miR-27a could play a vital role in CRC development and might contribute to a pathogenic mechanism. ${ }^{[1]}$ And in gastric cancer, Sun et al. found that a common polymorphism (rs895819) could act as a vital factor in cell susceptibility via modulating miR-27a and ZBTB10 levels.[2] And Renata Hezova et al. also indicated that in gastric cancer, it has been reported that the variant genotypes of rs895819 located at miR-27a conferred a $48 \%$ increased risk of developing gastric cancer in the Chinese population. Moreover, in this trend, age may play an important role. ${ }^{[3]}$ Similar to the study by
Renata Hezova et al., there was a finding also revealed in Chinese population, individuals with rs895819 G variants exhibited significantly increased risk of gastric cancer. Besides this, it was also reported that subjects with rs11671784 A variants experienced opposite biological effects. ${ }^{[4]}$ Furthermore, Yang et al. also indicated that the G/A polymorphism in miR-27a gene (rs11671784) decreased miR-27a expression and reduced gastric cancer risk. ${ }^{[5]}$ And in NSCLC, there was a study proved this SNP (rs895819) was an independently unfavorable factor for the prognosis of NSCLC. The authors demonstrated that the variant allele $G$ of rs895819 within pre-miR-27a was associated with a significantly increased risk of deaths for NSCLC. Moreover, they also found a significantly decreased response rate to platinum-based chemotherapy in advanced NSCLC patients with AG/GG genotype.[6] According to the study of Ma et al, allele G, allele G carrier (AG/GG) and genotype GG of rs895819 could be used as susceptible factors in NSCLC. Furthermore, they also reported that rs895819 was strongly associated with NSCLC carcinogenesis, but not with NSCLC progression in Chinese Han population. ${ }^{[7]}$ Similarly, in younger Chinese populations, Zhang et al. found that the SNP rs895819 may act as a risk factor for breast cancer. ${ }^{[8]}$ However it has been demonstrated that $G$ allele of rs895819 was also related to reduced German familial breast cancer risk. ${ }^{[9]}$ In addition, Yang et al. also found that the G-allele of rs895819, located in the terminal loop of the pre-miR27a oncogene, could reduce familial breast cancer risk strongly.[9] But interestingly, the study by Irene Catucci Paolo et al. revealed that the $G$ allele of rs895819 could not enhance or decrease risk of breast cancer in the Italian population. There was no specific association in them, ${ }^{10]}$ though the specific reason has been revealed now. Apart from acting a risk in many tumors, there were studies indicating it could play a protective role in the development of human cancer. For example, Didier Meulendijks et al. reported that Rs895819 in miR-27a improved the predictive value of DPYD variants to identify patients at risk of severe fluoropyrimidine-associated toxicity. However, the association between rs11671784 and fluoropyrimidine-associated toxicity was not quite clear.[11] In southern Chinese women, miR-27a rs895819 polymorphism could reduce risk of cervical cancer, especially $\mathrm{T}$ allele and CT or TT genotype, compared with C and CC respectively. ${ }^{[12]}$ In addition to rs895819 polymorphism, it has been reported that compared to A allele, rs11671784 $\mathrm{G}$ allele has significantly stronger effect in promoting chemo-sensitivity in bladder cancer. Furthermore, RUNX-1, a novel direct target of miR-27a, could play a vital role in the regulation of 
chemo-sensitivity in bladder cancer.[13] Not only in chemo-sensitivity, the study by Shi et al. showed that compared with AA homozygote, patients with AG/GG genotype could decrease susceptibility significantly in RCC cell. In addition, they also showed that rs895819 was associated with age and hypertension. Furthermore, the miR-27a polymorphism was found not to affect the survival of RCC cells in their study.[14] To estimate the clear association between the polymorphism and cancer, Wang et al. conducted a meta-analysis. They showed that the hsa-miR-27a rs895819 polymorphism did not correlate with overall cancer risk in the general population, however, the rs895819 AG genotype may play a protective role in the development of human cancer in white people.[15]

\section{MiR-27a in tumorigenesis}

MiR-27a is involved in many processes of tumor growth, including cell proliferation, apoptosis, migration, invasion, angiogenesis, and so on. Therefore, miR-27a may directly affect tumorigenesis. For example, Li et al. showed that over-expression of miR-27a inhibited the EGFR signaling pathway to alter the cell processes in 786-O cells, such as proliferation and apoptosis, migration and invasion, which acted as a vital role in RCC tumorigenesis.[16] Besides this, the assays have also demonstrated that no matter in vitro or in vivo, miR-27a could significantly decrease proliferation and invasion activities of ESCC cell in nude mice, by directly targeting KRAS to suppress tumorigenesis.[17] Moreover, $\mathrm{Li}$ et al. also reported that miR-27a could function as a suppressor in breast cancer. It targeted and down-regulated TMEM170B inhibiting the Wnt/ $\beta$-catenin pathway, which could suppress breast cancer proliferation, migration, and tumorigenesis. ${ }^{[18]}$ Apart from the studies above which suggesting miR-27a could play a vital role in repressing many tumors growth, there were evidences illustrating that it also acted as an enhancer in the angiogenesis of tumors. For example, TColangelo revealed that miR-27a, acted as an oncomiRNA from the early phases of colon tumorigenesis, modulating MHC class I surface exposure by directly targeting calreticulin which showing that miR-27a played a critical role in colon tumourigenesis. ${ }^{[19]}$ Similar to this, there was a study which proved that miR-23a/24-2/27a clusters could function as a causal role in mammary tumorigenesis and be an oncogene to enhance cell invasion and metastasis, through targeting Sprouty2 (SPRY2) and significantly activating p44/42 MAPK in mammary carcinoma.[20] In addition, recent studies demonstrated that miR-27a and EGFR functioned as downstream effector of mutant $\mathrm{p} 53$ and miR-27a/EGFR/ERK1/2 axis driven by $\mathrm{p} 53-273 \mathrm{H}$ could play a vital role in promoting human tumor formation. ${ }^{[21]}$ Furthermore, the study of Wang et al. also revealed that up-regulation of miR-27a could strongly enhance the malignant transformation of human bronchial epithelial cells, via targeting and negatively regulating Fbxw7, which suggested that miR-27a functioned as an oncogene in human tumorigenesis.[22] Similarly, B4GALT3, up-regulated by miR-27a, was recognized to significantly contribute to tumorigenic activities, like migration and invasion by $\beta 1$-integrin pathway.[23] In bladder urothelial carcinoma, $\mathrm{Xu}$ et al. reported that a new angiogenic factor AGGF1 was significantly lower in the high-grade urothelial carcinoma group than that in the low-grade urothelial carcinoma group or than that in the normal urothelium tissue group, compared with other angiogenic factors such as VEGFA. But surprisingly, over-expression of miR-27a suppressed the expression of AGGF1 which may promote tumorigenesis of cancer cells. [24]

\section{MiR-27a in cancer proliferation and apoptosis}

More and more studies have proved that miR-27a was associated with proliferation and apoptosis which were important parts of tumor cell growth. Therefore, by further understanding the mechanism it was promising to inhibit tumor occurrence. It was universally known that miR-27a played an important role in the cell proliferation and apoptosis. For example, Bao et al. described that in colorectal cancer, miR-27a could not only inhibit cell proliferation, enhance apoptosis, but also attenuate cancer cell migration via targeting SGPP1 and Smad2, which were inversely correlated with miR-27a. They also reported that down-regulation of p-STAT3 and up-regulation of cleaved caspase 3 also were linked to it and miR-27a was found significantly down-regulated. In vivo, miR-27a inhibited colon cancer cell growth in tumor-bearing mice. ${ }^{[25]}$ Similar to the study by Bao et al, miR-27a also had a positive effect on cell proliferation. It could function as a tumor suppressor in renal cell carcinoma by targeting epidermal growth factor receptor (EGFR), a direct target of miR-27a. Moreover, miR-27a could reduce its downstream gene cyclin D1, CDK2, and up-regulate the expression of the CDK inhibitor p27 to inhibit proliferation and induces apoptosis in 786-O cells. And in vivo, they found that miR-27a attenuated RCC tumor growth in mouse xeno-graft models.[16] Consistent with this discovery, miR-27a, significantly lower in ESCC cells, could inhibit esophageal squamous cell carcinoma cells proliferation via a direct target: KRAS.[17] Similar inhibitory effects of 
miR-27a on cell proliferation were found on gastric cancer cells. The study showed that in vitro and in vivo, down-regulation of miR-27a could inhibit proliferation of gastric cancer cells. It also reported that miR-27a, which was own-regulated, could significantly up-regulate the expression of p21; while in the expression of pglycoprotein as well as cyclin D1, it could exert opposite biological effects.[26] Furthermore, it was also reported that stable lentiviral transduction of anti-miRNAs targeted miR-27a in U87 glioma cells, and anti-miR-27a reduced the viability as well as invasiveness, and increased apoptosis of U87 cells. [27] In glioblastoma cell lines, miR-27a antagonists reduce cell proliferation and invasion. ${ }^{[28]}$ However, recent reports have shown that miR-27a promoted cell proliferation in GBM cell lines by directly targeting MXI1.[29, 30] Apart from these studies, Acunzo et al. reported that MET and EGFR in NSCLC could be regulated by miR-27a.[31] Furthermore, Yang et al. found that genistein activated miR-27a expression of NSCLC to induce apoptotic activities of cancer cell. And genistein may decrease MET protein expression levels in human lung cancer. ${ }^{[32]}$ It was reported that in oral oquamous cell carcinoma (OSCC), miR-27a, negatively correlated with the MCPH1 protein level, could target MCPH1 to induce cell apoptosis.[33] $\mathrm{Li}$ et al. also further confirmed the inhibitory effect of miR-27a on the proliferation. They revealed that miR-27a could target and down-regulate TMEM170B to inhibit the Wnt/ $\beta$-catenin pathway to suppress breast cancer proliferation. ${ }^{18]}$ Apart from inhibiting cancer cell proliferation activities, it was also found that miR-27a could play a crucial role in multiple tumors. Different from breast cancer above, in liver cancer, it was showed that miR-27a, which was up-regulated in obese patients, could promote proliferation ability of liver cancer cell through FOXO1. Meanwhile, miR-27a could enhance the G1/S cell cycle transition by significantly decreasing the cell cycle inhibitors p21 and p27, as well as increasing cell cycle regulator cyclin D1.[34] In addition, similar effects of miR-27a on HCC cell proliferation were also found by Li et al. They reported that miR-27a could be up-regulated significantly in hepatocellular carcinoma tissues and cell lines, and further demonstrated down-regulation of miR-27a inhibited HCC cell proliferation through promoting apoptosis and inducing G1-phase cell cycle arrest. Furthermore, they also reported that miR-27a promoted proliferation of hepatocellular carcinoma cell by suppressing target gene peroxisome proliferatoractivated receptor $\gamma^{.}{ }^{[35]}$ Interestingly, different from the study of Bao et al., there was a finding that reported the opposite result that up-regulation of miR-27a may play an important role in enhancing the proliferation and invasion of colon cancer cells, though the specific mechanisms were not quite clear in this finding. [36] Similar to it, miR-27a was shown to be oncogenic according to the study of Sudhakar Chintharlapalli et al. CDODA-Me, could decrease expression of miR-27a, resulting in enhanced expression of ZBTB10 and Myt-1 which subsequently induced downstream growth inhibitory, proapoptotic and antiangiogenic genes and pathways in colon cancer cells.[37] The same effect could be found in prostate cancer (PCa), over-expression of miR-27a resulted in significantly increased expression of androgen-regulated genes. In addition, miR-27a, which was a target of PHB, increased AR activity in PCa cells through loss of PHB corepressive activity to alter PCa cell growth. ${ }^{[38]} \mathrm{Li}$ et al. described that miR-27a could function as a tumor suppressor in renal cell carcinoma, but Peng et al. had a different view. They revealed that in vitro, over-expression of miR-27a could enhance cell proliferation and its low expression exerted opposite effects, while the direct target was unknown yet. Interestingly, they also reported that miR-27a was up-regulated in RCC tissues but had no effect on cell apoptosis. But the specific underlying mechanisms were again not quite clear in this study. ${ }^{[39]}$ It was reported that miR-27a could inhibit cell proliferation activity in ESCC cell, but there was a study where it exerted the opposite effects. MiR-27a-3p, over-expressed in ESCC cell lines and tissues, could enhance ESCC cell proliferation activity through targeting FBXW7. ${ }^{[40]}$ And the finding by Mónica Rivera-Díaz et al. revealed the FBXW7 and ADORA2B may be regulated by miR-27a in GBM patients, but these hypotheses in this study may need many investigations to prove them. Apart from these studies above, Wang et al. also revealed that up-regulation of miR-27a could not only enhance the malignant transformation but also promote cell proliferation activity of human bronchial epithelial cells induced by SV40 small T antigen, via targeting and negatively regulating Fbxw7.[22] (Figure 1)

\section{MiR-27a in cancer invasion and migration}

Metastasis, the most important step in tumor progression, has been a heavy burden to people all over the world. Numerous studies have proved that miR-27a was closely related to tumor metastasis. Accordingly, the specific metastasis process was urgently needed to be studied. Recently, there was emerging evidence that it could play a vital role in cell migration of many tumors. The study of Bao et al. has revealed miR-27a acted as a tumor suppressor by targeting SGPP1 and Smad2 to attenuate cell migration in colorectal cancer.[25] It was shown that miR-27a-3p transfection could significantly decrease 
the invasive ability of OSCC cell lines and YAP1 (Yes-associated protein-1) was a direct target gene of miR-27a-3p. Moreover, increasing the expression of miR-27a-3p could significantly decrease the expression level of YAP1 and several epithelial to mesenchymal transition (EMT) related molecules in OSCC cell lines, including Twist and Snail.[41] Similar effects could be found in prostate cancer. COUP-TFII was negatively regulated by miR-101 and miR-27a, and loss of miRNA promoted metastasis by inducing COUP-TFII. Moreover, FOXM1 and CENPF could be induced by COUP-TFII to promote metastasis.[42] These inhibitory effects on migration and invasion were revealed in oral squamous cell carcinoma (OSCC); a study showed that miR-27a, negatively correlated with the MCPH1 protein level, could target MCPH1 inhibiting cell invasion. ${ }^{[33]}$ Consistent with this discovery, there was a study that revealed miR-27a was down-regulated and could inhibit cell invasion by directly targeting and negatively regulating KRAS in esophageal squamous cell carcinoma cells.[17] In addition, over-expression of miR-27a functioned as an inhibition role in RCC cell by targeting epidermal growth factor receptor (EGFR). ${ }^{[16]}$ Similar to the studies above, Li et al. also reported that miR-27a was characteristic as a tumor suppressor by targeting and down-regulating TMEM170B to inhibit the Wnt/ $\beta$-catenin pathway, which could repress migration and tumorigenesis activities in breast cancer cell.[18] Except for the inhibitory effects on migration and invasion, miR-27a could act in an opposite role in many tumors. Sun et al. has showed that miR-27a, which was up-regulated, could target and up-regulate B4GALT3 to promote migration, invasion by $\beta 1$-integrin pathway and regulate EMT-associated molecules. Meanwhile, instead of the stability of B4GALT3 miRNA and protein, miR-27a increased the transcription of B4GALT3. They also detected the effects induced by miR-27a could be abolished via knock-downing B4GALT3 in cervical cancer cells.[23]
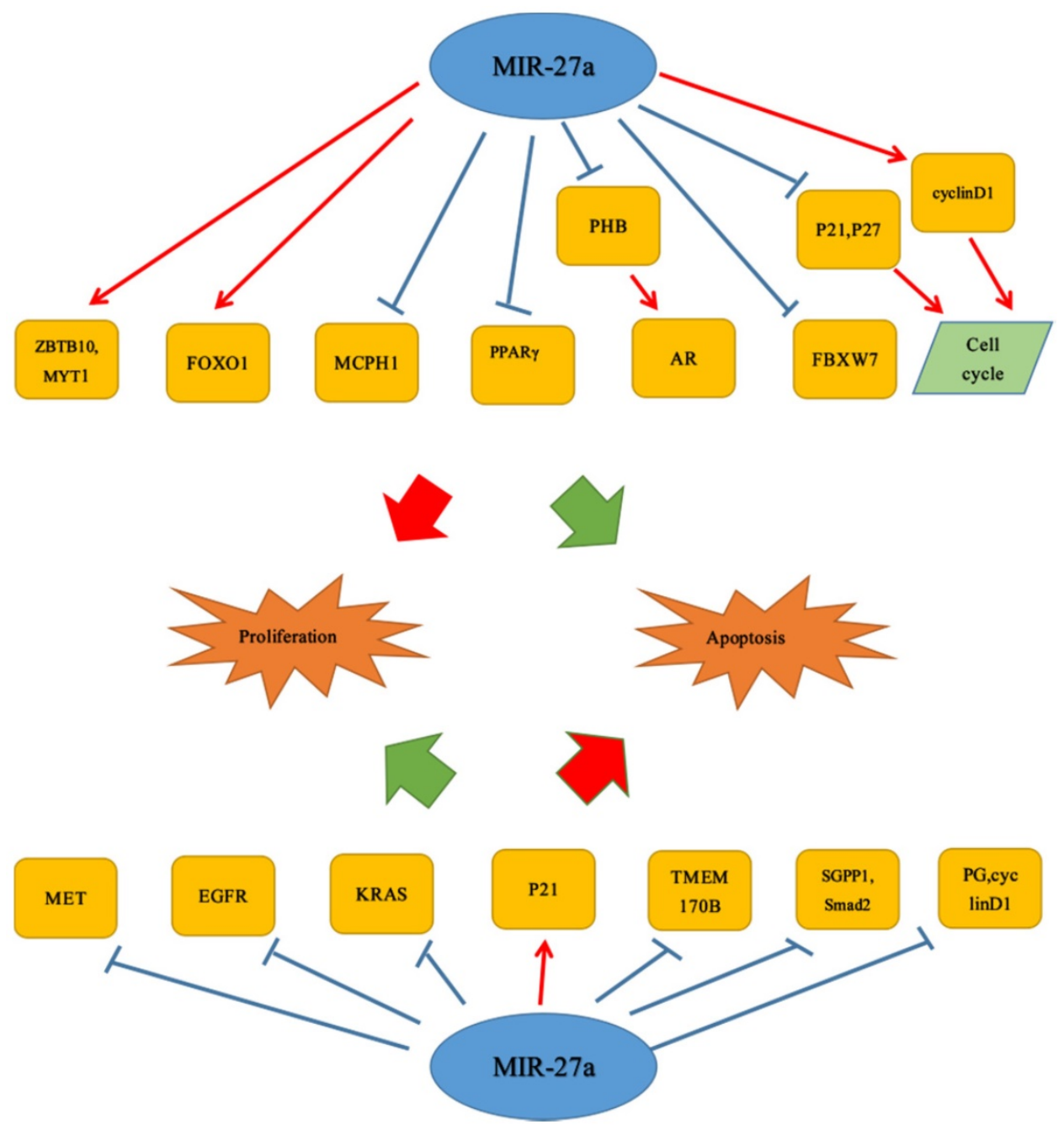


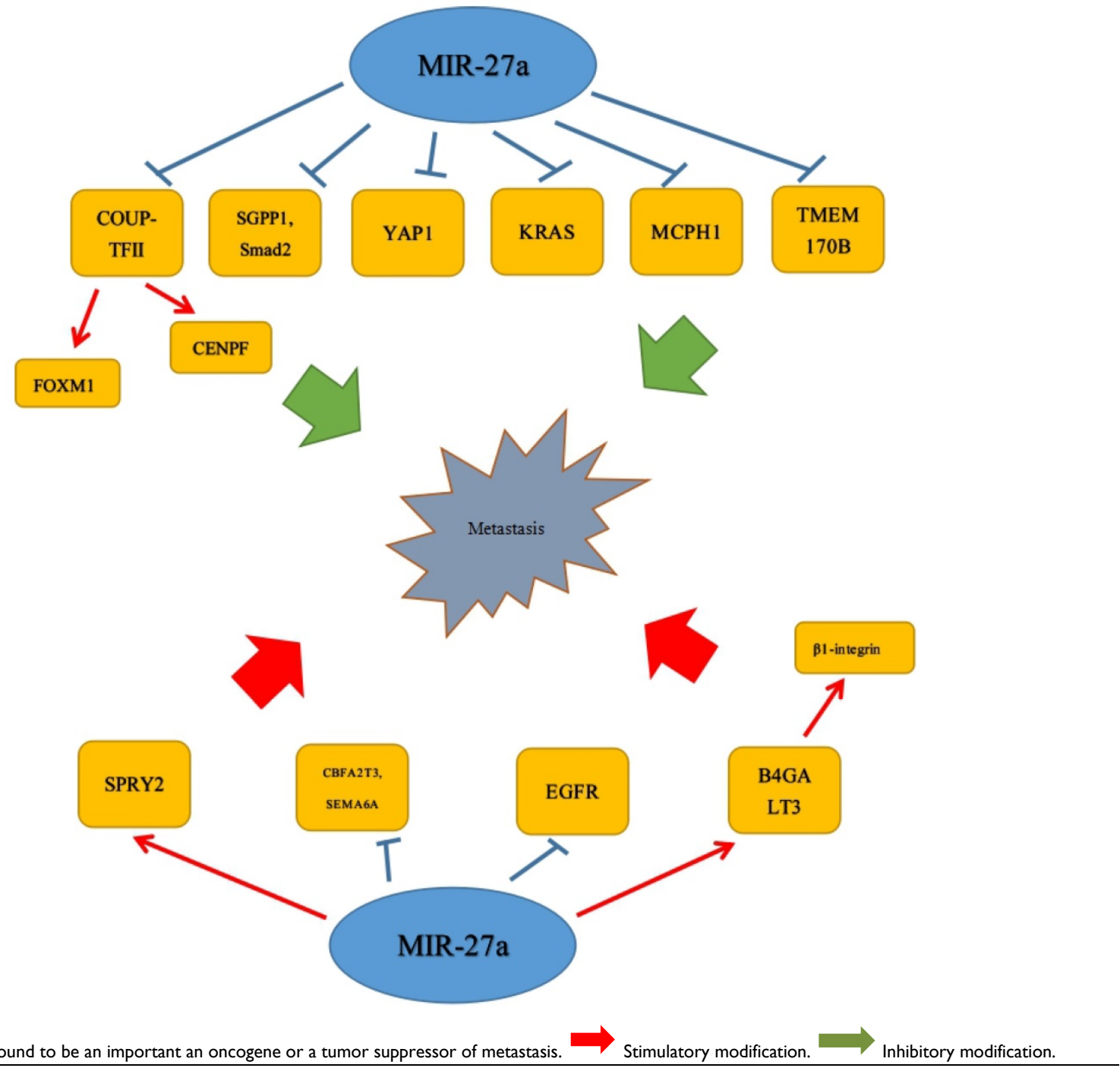

Similarly, it was reported that in osteosarcoma, CBFA2T3, which was negatively related to miR-27a expression, was a target of miR-27a, and miR-27a co-over-expression promoted metastatic properties of SAOS2. Furthermore, miR-27a inactivation by sponges reduced metastatic properties in HOS and KHOS osteosarcoma cells. ${ }^{[43]}$ At the same time, miR-27a could regulate other OS-related genes, including SEMA6A, which was an established miR-27a/b target. ${ }^{[44]}$ Moreover, the expression of SEMA6A was negatively associated with miR-27a and the metastatic potential in SAOS2 and LM7 cells. In addition to studies above, an article also revealed that miR-23a/24-2/27a cluster, which was increased in mammary carcinoma, could promote distant cell migration and invasion in mammary carcinoma through SPRY2 in vitro. ${ }^{[20]}$ Furthermore, SPRY2, which was directly targeted by the miR-23a/24-2/27a cluster, was recognized as an inhibitor of the p44/42 MAPK signaling pathway, ${ }^{[45,46]}$ and $\mathrm{p} 44 / 42$ MAPK enhanced breast cancer cell (MCF-7) migration and invasion. ${ }^{47,48]}$ EGF regulated the expression of miR-23a/24-2/27a cluster partly via c-MYC, which played an important role in cell migration activity. Interestingly, different from $\mathrm{Li}$ et al, the Transwell chamber with Matrigel assay by Peng et al. demonstrated that high levels of miR-27a strongly promoted the invasion of RCC cells, whereas low expression of miR-27a had an opposite effect.[39] The results from the Matrigel Transwell assay also indicated that the inhibition of miR-27a substantially impaired the invasiveness of U87 cells, though the specific underlying mechanisms was not quite clear in this study.[27] (Figure 2)

\section{MiR-27a in angiogenesis}

Angiogenesis could significantly promote cell growth and was generally accepted as a vital characteristic in various malignant neoplasms. Increasing numbers of studies revealed that miR-27a played a crucial role in enhancing or inhibiting the tumor angiogenesis. It has been reported that in 
animal models, up-regulation of miR-27a may promote angiogenesis in tumor tissues. However, the specific mechanisms by which it acted were still unclear. ${ }^{[49]}$ Tang et al. also demonstrated that miR-27a could lead to angiogenesis through mediating endothelial differentiation of BCSLCs. Hence, it could be a novel target for anti-angiogenesis cancer therapy.[50] Moreover, $\mathrm{Li}$ et al reported that it determined endothelial differentiation of breast cancer stem cells, contributing to tumor neoangiogenesis. ${ }^{[51]}$ But the specific target gene has been not shown. Several findings revealed that angiogenesis could be promoted by miR-27a/b through directly targeting the angiogenesis inhibitor SEMA6A, which acted as inhibitors of angiogenesis.[52, 53] Over-expression of miR-27a/b enhanced angiogenesis in vitro, whereas inhibition of $\mathrm{miR}-27 \mathrm{a} / \mathrm{b}$ reduced angiogenesis in vitro and in vivo. [44] These data were in accordance with the study by Zhou et al., demonstrating that the miR-23/27/24 clusters could regulate angiogenesis. ${ }^{[54]}$ That MiR-27a functioned as an angiogenesis promoter has been confirmed by Jennifer A. Young et al. MiR-27a targeted VE-cadherin to be a major regulator of angiogenesis and regulated EC junctions to control vascular integrity. And they also reported miR-27a was rapidly down-regulated with the stimulation of in vitro angiogenesis, and the expression was reduced

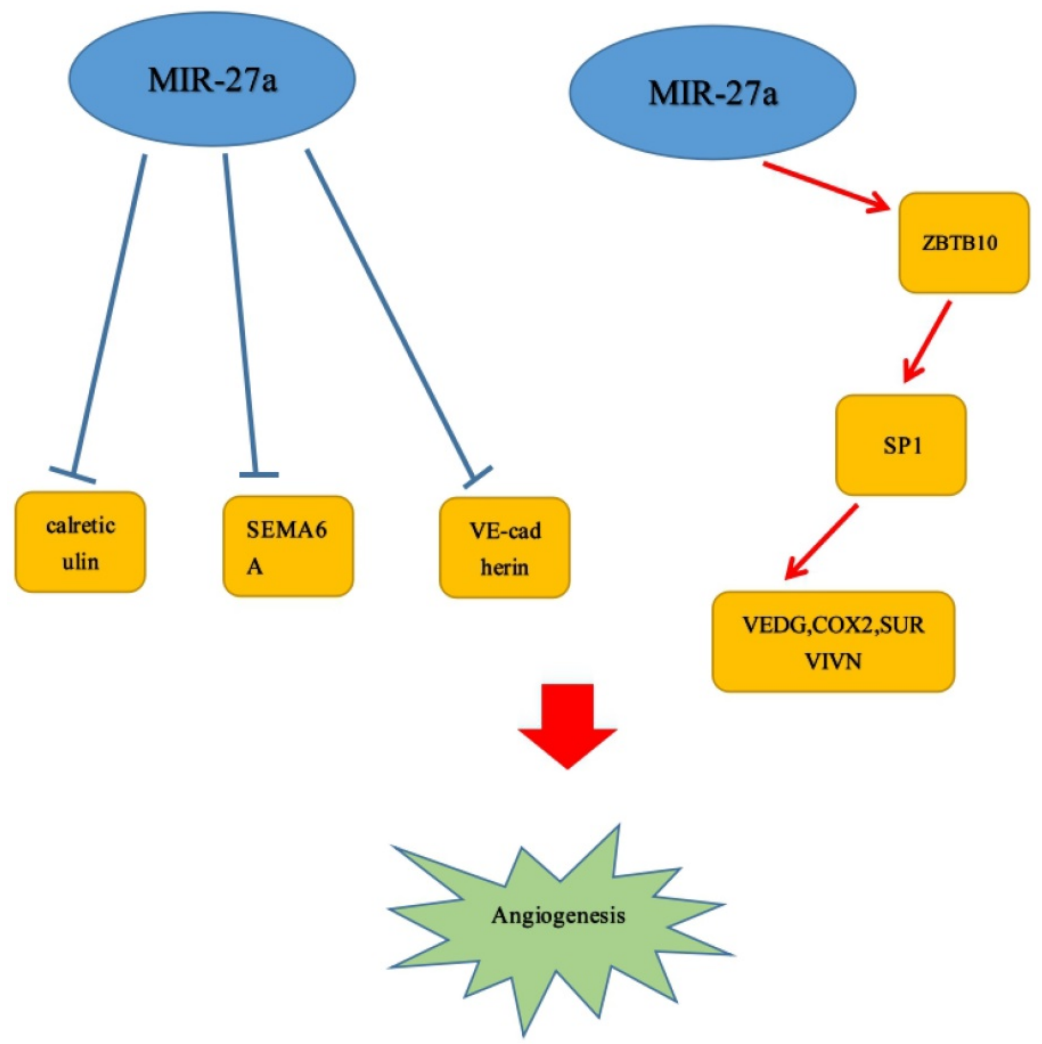

Figure 3. MiR-27a could promote the angiogenesis of several tumors. in neovessels in vivo.[55] Similarly, in the study of Zhao et al., miR-27a-3p deregulation could contribute to vasculogenic mimicry (VM) and metastasis by directly targeting VE-cadherin. ${ }^{[56]}$ Consistent with this discovery, there was a study also reported the evidence that microRNA-27a: ZBTB10-specificity protein pathway could lead to breast cancer angiogenesis. ${ }^{[57]}$ In addition, Lai et al. found that FSH may enhance not only VEGF but also Cox2 and survivin expression via activating miR-27a: ZBTB10-Sp1 signal pathway. Next, it further induced angiogenesis in ovarian tumor. And VEGF, Cox2 have been reported that they acted as factors to contribute to tumor angiogenesis. ${ }^{[58]}$ Interestingly, in previous studies, a finding has reported that FSH could also enhance VEGF expression mediated by survivin and HIF1 a by PI3K/AKT signal pathway. ${ }^{[59]}$ Similar to the studies above, miR-27a, acted as an angiogenesis promoter, could directly target and down-regulate calreticulin affecting MHC class I exposure to affect cell proliferation and angiogenesis in vitro. ${ }^{[19]}$ (Figure 3)

\section{The molecular or cellular mechanisms of miR-27a}

MicroRNAs regulate gene expression at the post-transcriptional level by binding to the partial sequence homology of the 3'-untranslated region of target messenger (m)RNA. In recent years, various studies have been reported that miR-27a could regulate other related molecules to affect tumorigenesis. MiR-27a affected tumor growth by targeting different genes or signaling pathways to affect their protein expression levels. It was involved in development processes of tumors, such as proliferation, apoptosis, invasion, migration and angiogenesis. For example, it could function as a tumor suppressor via targeting EGFR (a direct target of miR-27a). Besides this, it could reduce its downstream gene cyclin D1, CDK2, and up-regulate the expression of the CDK inhibitor p27 to inhibit proliferation. ${ }^{[16]}$ The molecular mechanism by which miR-27a targets different molecules has been demonstrated in various findings, but some specific mechanism in tumorigenesis are still unclear. The signaling pathway played an important role in the molecular mechanism of cancer development. MiR-27a could affect the p44/42 MAPK signaling pathway to influence migration activity. SPRY2 was a 
directly targeted of miR-27a and recognized as an inhibitor of the p44/42 MAPK signaling pathway, hence, miR-27a could target SPRY2 to regulate p44/42 MAPK signaling pathway. $[45,46]$

Epithelial mesenchymal transition (EMT) is a process where epithelial cells lose their cell polarity and cell-cell adhesion or lose typical epithelial features and are transformed into mesenchymal cells, and could play a critical role in cell migration. A number of studies have revealed that miR-27a could regulate the cell migration by epithelial mesenchymal transition. [23,41] Apart from this, miR-27a could also affect the cell cycle to regulate tumor cell activity. For example, miR-27a could enhance the G1/S cell cycle transition by significantly decreasing the cell cycle inhibitors p21 and p27, as well as increasing cell cycle regulator cyclin D1 to promote proliferation. ${ }^{34]}$ Additionally, down-regulation of miR-27a inhibited HCC cell proliferation through inducing G1-phase cell cycle arrest.[35] MiR-27a influenced the cell proliferation or migration or angiogenesis to further promote or inhibit the tumor growth.

\section{MiR-27a regulates intermolecular interaction}

P53, functioning as a tumor suppressor, has played a vital role for protection against cancer. Mutant p53 was different with p53 and promoted the tumor development. Mutant p53, targeting and down-regulating directly miR-27a, could bind to the miR-27a promoter region and repress the expression of miR-27a. And epidermal growth factor receptor (EGFR), a direct target of miR-27a, further promoted a sustained EGF-induced extracellular signal-regulated kinase $1 / 2$ activation to promote tumor development. ${ }^{[21]}$ MET, a membrane receptor for the hepatocyte growth factor (HGF), ${ }^{[60]}$ could significantly regulate the miR-23a/24-2/27a cluster in NSCLC cells. EGFR, as we know, was a membrane receptor and could enhance the growth epithelial tissues.[3-5, 61-63] And sprouty2 protein also increased the expression of MET and EGFR protein levels in the cells. ${ }^{[64-66]}$ MiR-27a, a member of miR-23a/24-2/27a cluster, directly targeted MET and EGFR to repress their levels. Apart from this, there was another way to regulate MET and EGFR. MiR-27a indirectly acted on their protein levels by targeting sprouty 2 to reduce the MET and EGFR levels in NSCLC. [31] Follicle-stimulating hormone (FSH) targeted miR-27a to up-regulate VEGF, Cox2 and survivin in ovarian cancer. And over-expression of Cox2, survivin and VEGF may promote the cancer angiogenesis. Sp1 acted as a vital role in microRNA-27a: ZBTB10 pathway, besides, ZBTB10 could target it to facilitate ovarian cancer angiogenesis.[10] Epidermal growth factor (EGF) regulated the expression of miR-23a/24-2/27a cluster partly through c-MYC.[20] And the miR-23a/24-2/27a cluster directly and negatively regulated SPRY2 to activate MAPK signaling to enhance breast cancer cell (MCF-7) migration and invasion. $\left.{ }^{47,} 48\right] 3$ acetyl-11-keto-bboswellic acid (AKBA) and curcumin repressed the expression of miR-27a in colorectal cancer. And miR-27a was down-regulated by curcumin treatment alone, but a combined treatment of AKBA and curcumin could further inhibit the level of miR-27a. Similarly, the combined treatment could increase the FBXW7 expression level. MiR-27a targeted directly FBXW7 to further inhibit cyclin D1 and cMyc.[67] The epithelial-mesenchymal transition (EMT), played a critical role in metastasis for cancer progression. The process where epithelial cells lose their cell polarity and cell-cell adhesion was called EMT which could be a critical step in the dissemination of malignant diseases. YAP1 (Yes-associated protein-1) was a direct target gene and negatively correlated with miR-27a. Besides, miR-27a-3p, as one of the vital upstream molecules, could influence EMT-related inducers Snail and Twist through YAP1-OCT4/Sox2 signal axis.[41] (Figure 4)

\section{Clinical significance of miR-27a}

\section{MiR-27a affects drug sensitivity and resistance}

As everyone knows, drug resistance is a great threat to cancer patients and a heavy burden to every family. Accordingly, it is urgent for us to understand the mechanism of drug resistance. Increasing studies demonstrated that miR-27a participated in the occurrence of cell drug resistance. MiR-27a could be a target of the drugs or target different genes to enhance or inhibit the drug sensitivity, hence, it may be a potential therapeutic target for the clinical therapy for tumors.

Down-regulation of miR-27a could not only significantly reduce the expression of P-glycoprotein and the transcriptional activity of cyclinD1 but also up-regulate the expression of p21 to repress cell vincristine, adriamycin and 5-fludrouracil drug resistance. ${ }^{[26]}$ Additionally, there was a study reported that miR-331-5p and miR-27a could increase the sensitivity of K562/DOX and HL60/DOX cells to DOX. The expression of miR-331-5p and miR-27a was negatively related to the expression of drug-resistant factors such as P-glycoprotein (P-gp) and MDR1. P-gp down-regulation could reverse the drug-resistance, and down-regulated MDR1 could increase sensitivity of the K562-resistant cancer cells to DOX.[68] Interestingly, it was also revealed that miR-27a and 


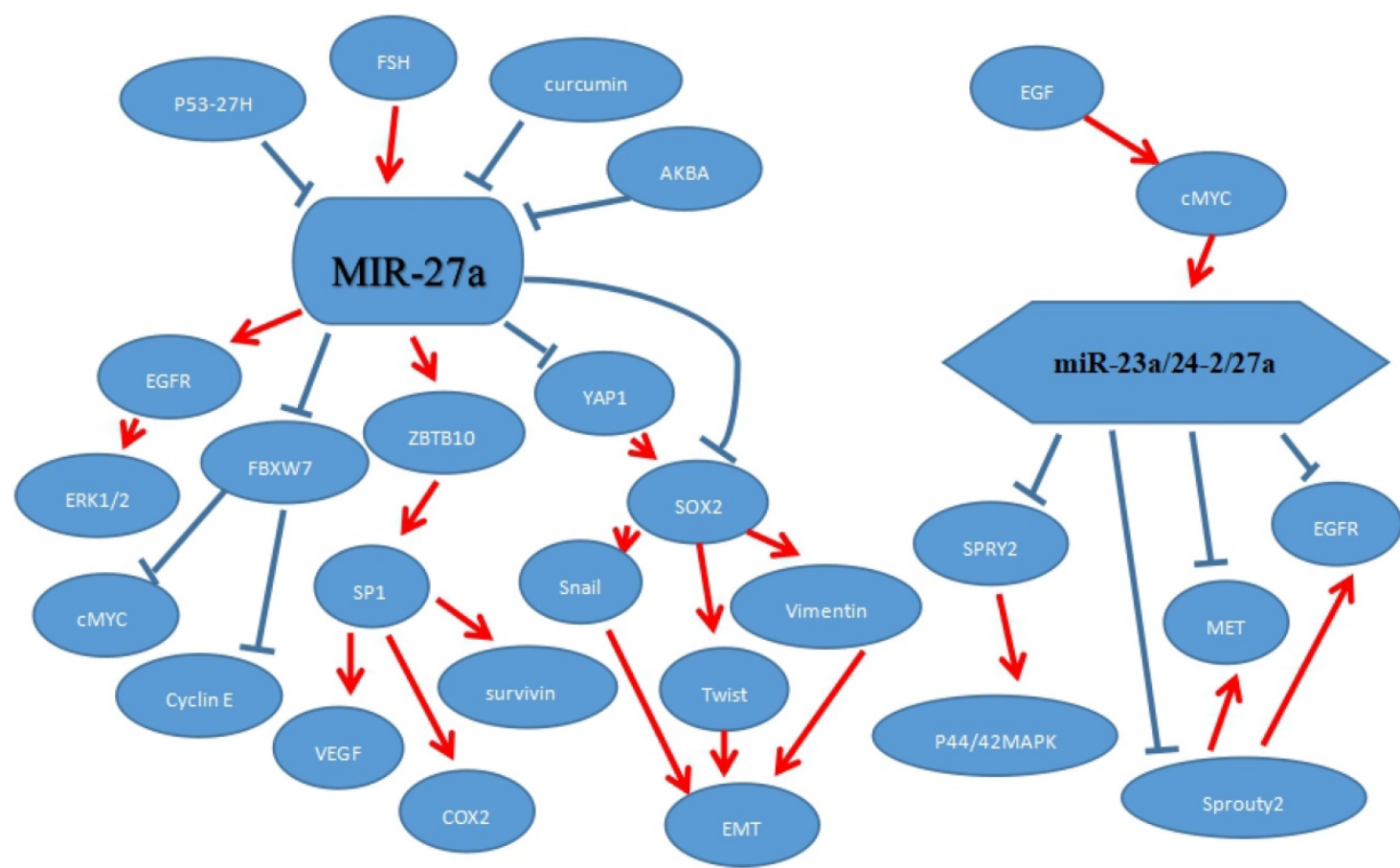

Figure 4. MiR-27a or miR-23a/24-2/27a regulated intermolecular interaction.

miR-451, being in up-regulated, could promote expression level of MDR1 in human ovarian cancer cell and cervix carcinoma cell.[69] Therefore, miR-27a may have a promising future in the treatment of DOX resistance. Similar to the effect of repressing the cell drug resistance, the gene of BAK may play a vital role of functional target of miR-27a in breast cancer. Moreover, knockdown of miR-27a increased the sensitivity of cancer cells to cisplatin treatment by BAK-SMAC/DIABLO-XIAP pathway. The effect of DOX, TRAIL (tumor necrosis factor-related apoptosis-inducing ligand (TRAIL)), and 5-FU to cancer could also be promoted by miR-27a. And there may exist a negative correlation between miR-27a and Bak in breast cancer. ${ }^{[70]}$ It was possible that GBM patients with high miR-27a expression levels may respond better to certain chemotherapeutic agents, but the specific underlying mechanisms were not quite clear in this study. Responding better, we know, may mean a better overall survival (OS). In addition, Deng et al. also found that RUNX-1 could act as a novel direct target of miR-27a, which also played a vital role in the regulation of chemo-sensitivity in bladder cancer. They also found that when compared to A allele, rs11671784 G allele has a significantly stronger effect in promoting chemo-sensitivity in bladder cancer partially through reducing P-gp expression. ${ }^{[13]}$

Apart from the role of inhibiting the cell drug resistance, miR-27a could exert an opposite effect in many tumors. Zhao et al. found that the down-regulation of miR-27a could repress the cell drug resistance in GC cells.[26] But interestingly, there was an opposite result. A study reported that HIF-1a and miR-27a were up-regulated, and HIF-1a acted as an up stream regulator of miR-27a to induce the expression of MDR1/P-gp, LRP and Bcl-2 in GC. And HIF-1a-miR-27a signaling enhanced the properties of MDR. [71] In recent years, studies have shown that miR-27a, as a member of the miRNA family, has been demonstrated to affect the MDR in GC cell significantly.[26] Different from the study by Deng et al., it has been proved that SLC7A11 expression was negatively correlated with miR-27a expression. And decreased expression of miR-27a could contribute to cell resistance of cisplatin through targeting the cystine/glutamate exchanger SLC7A11 in bladder cancer. Therefore, it may be promising for SLC7A11 inhibition with sulfasalazine to treat cisplatinresistant disease. ${ }^{[72]}$ In addition, Lin et al. showed that in prostate cancer, COUP-TFII was negatively regulated by miR-101 and miR-27a, and loss of miRNA promoted metastasis by inducing COUP-TFII. Moreover, FOXM1 and CENPF could be induced by COUP-TFII to promote enzalutamide resistance. Therefore, miR-101 or miR-27a would act as a promising target in therapy. ${ }^{[42]}$ Similar to studies above, miR-27a/b could convert NOF to CAF-like fibroblasts (miR-27a/b-induced CAF) and indirectly affect chemo-sensitivity of esophageal cancer cells. MiR-27a/b-induced CAF enhanced resistance to cisplatin and high serum expression of miR-27a/b 
was correlated negatively with the response to chemotherapy. ${ }^{[73]}$ (Figure 5)

\section{MiR-27a affects treatment of tumor}

MiR-27a could regulate different molecules to affect the tumor development, and in the therapy of cancer, it also played an important role. As a vital emerging modulator in molecular or cellular pathways, miR-27a could be a target or target related genes to inhibit the tumor cell growth. MiR-27a acted as a promising target and many cancer patients could be benefited by it. Now, we briefly cite examples of cancer treatment through different signaling pathways.
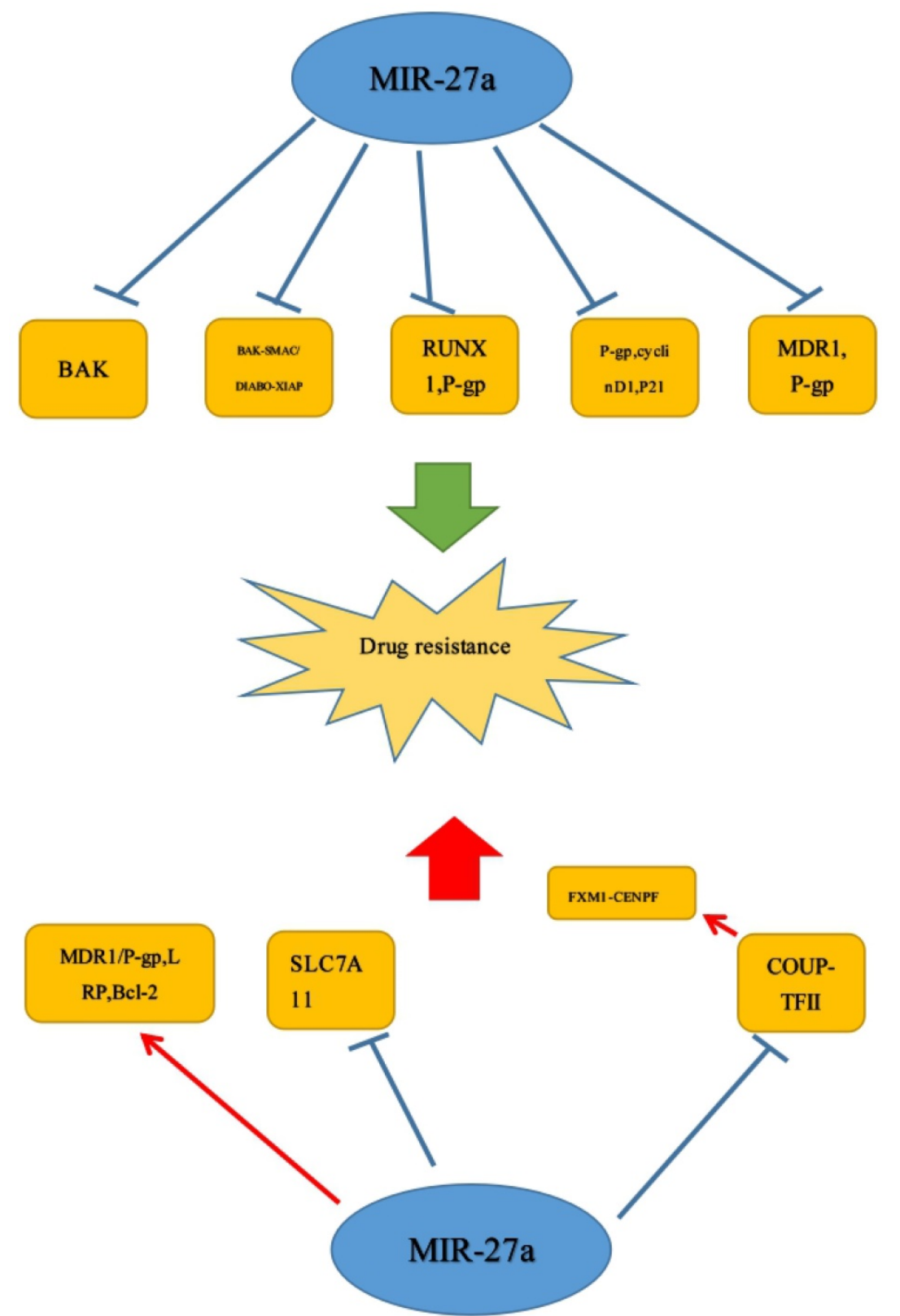

Figure 5. MiR-27a was confirmed to increase or decrease drug sensitivity in human cancer, which could be a target for the cancer therapy. Stimulatory modification.
Prostate cancer $(\mathrm{PCa})$, the most commonly diagnosed malignancy among males, could be a significant threat and lead to a severe rate of cancer death all the world. MiR-27a may be a potential target for the treatment of prostate cancer and provide the patients with more benefits. In the cancer development stages or types, androgen receptor (AR) may act as a vital role in prostate cancer. ${ }^{[74]} \mathrm{MiR}-27 \mathrm{a}$, a vital target of AR, could down-regulate mitogen-activated protein kinase kinase 4(MAP2K4) to mediate prostate cancer progression. MAP2K4, as we know, was a member of mitogen-activated protein kinase (MAPK) family and involved in a wide variety of cellular processes. MiR-27a could function as an oncogene to target MAPK4 for therapy of prostate cancer. MiR-27a was down-regulated in prostate cancer, and dihydrotestosterone (DHT) promoted the expression of miR-27a via AR signaling to decrease prostate cancer cell proliferation and migration. Besides, PI3K/Akt signaling also played a vital role in the development of prostate cancer. It could down-regulate miR-27a, furthermore, the miR-27a targeting MAP2K4 to affect the tumor growth. LY294002, acting as an inhibitor of PI3K/Akt signaling pathway, could directly affect the cancer progression.The two signaling above(AR signaling and PI3K/Akt signaling), targeted miR-27a to inhibit tumor progression. ${ }^{[75](F i g u r e ~ 6) ~}$

Breast cancer is a major progressive health threat with the highest incidence and mortality in females all around the world. In recent years, many reports have demonstrated that breast cancer was a biologically heterogeneous disease, ${ }^{[76,}$ 77] therefore, cellular pathways could be a key role in the treatment of cancer. Arsenic trioxide (ATO), as we know, in the treatment of APL (acute promyelocytic leukemia), could play a vital role and have a better clinical effect. It may also be a novel anti-tumor drug in breast cancer patients. ATO could directly target and down-regulate miR-27a to affect breast cancer cell development. Fbw7, acted as a tumor suppressor, and could be a direct target of miR-27a in breast cancer in various studies. ${ }^{[22,78,79]}$ It was 
down-regulated by miR-27a to suppress the breast cancer cells growth. Hence, in the treatment of breast cancer, miR-27a could be a promising target and affect cell activities. ${ }^{[80]}$ (Figure 6)

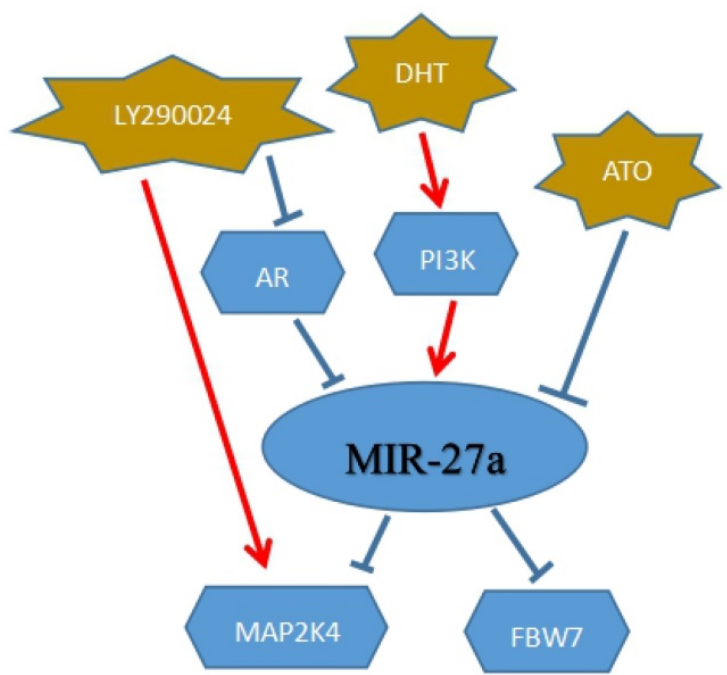

Figure 6. MiR-27a could be a target to the clinical therapy of cancer. DHT: dihydrotestosterone; ATO: Arsenic trioxide

\section{MiR-27a reflects the cancer prognosis}

The expression level of miR-27a was different in various tumors and it could be a target of the antineoplastic drugs or could directly/indirectly target genes to promote or inhibit the tumorigenesis. In the drug resistance or therapy of the cancer, miR-27a has been played a vital role and significantly affects the patient's prognosis. Accordingly, miR-27a may be a potential biomarker for cancer patients' prognoses. The expression level of a combination of miR-27a or miR-331-5p could suggest clinical relapse or poor prognosis. For example, the patients with relapsed lymphocytic leukaemia or myeloid leukaemia, the expression of miR-27a or miR-331-5p was low but untreated patients was high. ${ }^{[26]}$ Apart from it, it was possible that GBM patients with high miR-27a expression levels may respond better to certain chemotherapeutic agents. And the study also revealed that in GBM patients whose miR-27a expression levels were high would have a better outcome, while the GBM patients whose miR-27a expression levels were low may have a poor prognosis. Similarly, in the patients with metastatic or recurrent gastric cancer, miR-27a expression could be an independent predictive factor for OS. The patients whose miR-27a expression was higher would lead to poor outcomes. ${ }^{[81]}$ The obese liver cancer patients with high miR-27a levels would tend to promote liver cancer proliferation when compared to those with low miR-27a levels. Hence, miR-27a may suggest that the obese liver cancer patients may have poor prognosis in the future.[34] Tang et al. reported that the osteosarcoma patients could increase the levels of miR-27a, when compared with healthy people. Additionally, the patients with advanced clinical stage, positive distant metastasis and poor response to chemotherapy tended to have high expression serum levels of miR-27a. Furthermore, they found that miR-27a could act as an prognostic factor to predict overall survival and disease-free survival..82] Accordingly, these results above indicated that miR-27a may have a promising prognostic value of osteosarcoma patients prognosis. TMEM170B could be a targeted and down-regulated directly by miR-27a in breast cancer. Moreover, the Wnt/ $\beta$-catenin pathway could be repressed by TMEM170B to suppress breast cancer progression. Breast cancer patients whose TMEM170B expression was lower had a shorter OS time, and ones whose $\beta$-catenin expression was higher could reduce the OS time. Furthermore, the lower expression of TMEM170B was significantly related to the pathological stage and the TNM status. ${ }^{[18]}$

\section{Conclusions}

In this review, we focused on the function of miR-27a in tumor biology and clinical significance. It could play a vital role in the progression of cancers. In tumor biology, it could have the polymorphisms and significantly influence tumor cells tumorigenesis, proliferation, apoptosis, invasion, migration and angiogenesis. And in clinical significance, miR-27a could affect clinical therapy, drug sensitivity of patients and patient's prognosis. In detail, miR-27a was associated with various target genes to regulate tumor growth, like MCPH1, MXI1, FBXW7, KRAS, $\mathrm{PHB}$, and so on. In conclusion, miR-27a participated in tumor biology and affected the clinical treatment, therefore, we suggested that miR-27a acted as a promising biomarker and potential therapeutic target in various tumors.

\section{Abbreviations}

FSH: Follicle-stimulating hormone; AKBA: 3 acetyl-11-keto-b-boswellic acid; EMT: epithelialmesenchymal transition; NSCLC: nonsmall-cell lung cancer; EGFR: epidermal growth factor receptor; RCC: renal cell carcinoma; OSCC: oral squamous cell carcinoma; HCC: hepatocellular cancer; DOX: doxorubicin; GC: gastric cancer; GBM: glioblastoma; PCa: prostate cancer; VEGF: vascular endothelial growth factor; OS: overall survival; ESCC: esophageal squamous cell carcinoma; CRC: colorectal cancer; SNPs: Single-nucleotide polymorphisms; MDR: multidrug resistance; CDK: cyclin-dependent kinase; AR: androgen receptor; MAP2K4: mitogen-activated 
protein kinase kinase 4; DHT: dihydrotestosterone; ATO: Arsenic trioxide; APL: acute promyelocytic leukemia.

\section{Acknowledgements}

This research was supported by the National Key Research and Development Program of China (No. 2016YFC0905900), National Natural Science Foundation of China (No. 81872365), the "333" Talent Project of Jiangsu Province [No. 4(2016)] and Innovation Team Construction Project of Nanjing Medical University (No. JX102GSP201727).

\section{Competing Interests}

The authors have declared that no competing interest exists.

\section{References}

1. Cao Y, Hu J, Fang Y, et al. Association between a functional variant in microrna-27a and susceptibility to colorectal cancer in a chinese han population. Genet Mol Res. 2014; 13: 7420-7.

2. Sun $Q, G u H$, Zeng $Y$, et al. Hsa-mir-27a genetic variant contributes to gastric cancer susceptibility through affecting mir-27a and target gene expression. Cancer Sci. 2010; 101: 2241-7.

3. Hezova R, Kovarikova A, Bienertova-Vasku J, et al. Evaluation of snps in mir-196-a2, mir-27a and mir-146a as risk factors of colorectal cancer. World J Gastroenterol. 2012: 18: 2827-31.

4. Song B, Yan G, Hao H, et al. Rs11671784 g/a and rs895819 a/g polymorphisms inversely affect gastric cancer susceptibility and mir-27a expression in a chinese population. Med Sci Monit. 2014; 20: 2318-26.

5. Yang Q, Jie Z, Ye S, et al. Genetic variations in mir-27a gene decrease mature mir-27a level and reduce gastric cancer susceptibility. Oncogene. 2014; 33: 193-202.

6. $\mathrm{Xu} \mathrm{J}$, Yin $\mathrm{Z}$, Shen $\mathrm{H}$, et al. A genetic polymorphism in pre-mir-27a confers clinical outcome of non-small cell lung cancer in a chinese population. PLoS One. 2013; 8: e79135

7. Ma J-Y, Yan H-J, Yang Z-H, et al. Rs895819 within mir-27a might be involved in development of non small cell lung cancer in the chinese han population. Asian Pacific Journal of Cancer Prevention. 2015; 16: 1939-44.

8. Zhang $\mathrm{N}$, Huo $\mathrm{Q}$, Wang $\mathrm{X}$, et al. A genetic variant in pre-mir-27a is associated with a reduced breast cancer risk in younger chinese population. Gene. 2013; 529: 125-30

9. Yang $R$, Schlehe $B$, Hemminki $K$, et al. A genetic variant in the pre-mir-27a oncogene is associated with a reduced familial breast cancer risk. Breast Cancer Res Treat. 2010; 121: 693-702.

10. Catucci I, Verderio P, Pizzamiglio S, et al. The snp rs895819 in mir-27a is not associated with familial breast cancer risk in italians. Breast Cancer Res Treat. 2012; 133: 805-7.

11. Meulendijks D, Henricks LM, Amstutz U, et al. Rs895819 in mir27a improves the predictive value of dpyd variants to identify patients at risk of severe fluoropyrimidine-associated toxicity. Int J Cancer. 2016; 138: 2752-61.

12. Xiong $\mathrm{XD}$, Luo $\mathrm{XP}$, Cheng J, et al. A genetic variant in pre-mir-27a is associated with a reduced cervical cancer risk in southern chinese women. Gynecol Oncol. 2014; 132: 450-4.

13. Deng $\mathrm{Y}$, Bai $\mathrm{H}, \mathrm{Hu} \mathrm{H}$. Rs11671784 g/a variation in mir-27a decreases chemo-sensitivity of bladder cancer by decreasing mir-27a and increasing the target runx-1 expression. Biochem Biophys Res Commun. 2015; 458: 321-7.

14. Shi $\mathrm{D}, \mathrm{Li} \mathrm{P}, \mathrm{Ma} \mathrm{L}$, et al. A genetic variant in pre-mir-27a is associated with a reduced renal cell cancer risk in a chinese population. PLoS One. 2012; 7: e46566.

15. Wang Z, Lai J, Wang $Y$, et al. The hsa-mir-27a rs895819 (a>g) polymorphism and cancer susceptibility. Gene. 2013; 521: 87-90.

16. Li Y, Li J, Sun X, et al. Microrna-27a functions as a tumor suppressor in renal cell carcinoma by targeting epidermal growth factor receptor. Oncol Lett. 2016; 11: 4217-23.

17. Jiang Y, Duan Y, Zhou H. Microrna-27a directly targets kras to inhibit cell proliferation in esophageal squamous cell carcinoma. Oncol Lett. 2015; 9: 471-7.

18. Li M, Han $\mathrm{Y}$, Zhou $\mathrm{H}$, et al. Transmembrane protein $170 \mathrm{~b}$ is a novel breast tumorigenesis suppressor gene that inhibits the wnt/beta-catenin pathway. Cell Death Dis. 2018; 9: 91.

19. Colangelo T, Polcaro G, Ziccardi P, et al. Proteomic screening identifies calreticulin as a mir-27a direct target repressing mhc class i cell surface exposure in colorectal cancer. Cell Death Dis. 2016; 7: e2120.
20. Li X, Liu X, Xu W, et al. C-myc-regulated mir-23a/24-2/27a cluster promotes mammary carcinoma cell invasion and hepatic metastasis by targeting sprouty2. J Biol Chem. 2013; 288: 18121-33.

21. Wang W, Cheng B, Miao L, et al. Mutant p53-r273h gains new function in sustained activation of egfr signaling via suppressing mir-27a expression. Cell Death Dis. 2013; 4: e574.

22. Wang $\mathrm{Q}, \mathrm{Li} \mathrm{DC}, \mathrm{Li} \mathrm{ZF}$, et al. Upregulation of mir-27a contributes to the malignant transformation of human bronchial epithelial cells induced by sv 40 small $t$ antigen. Oncogene. 2011; 30: 3875-86.

23. Sun $Y$, Yang X, Liu M, et al. B4galt3 up-regulation by mir-27a contributes to the oncogenic activity in human cervical cancer cells. Cancer Lett. 2016; 375: 284-92.

24. Xu Y, Zhou M, Wang J, et al. Role of microrna-27a in down-regulation of angiogenic factor aggf1 under hypoxia associated with high-grade bladder urothelial carcinoma. Biochim Biophys Acta. 2014; 1842: 712-25.

25. Bao Y, Chen Z, Guo Y, et al. Tumor suppressor microrna-27a in colorectal carcinogenesis and progression by targeting sgpp1 and smad2. PLoS One. 2014; 9: e105991.

26. Zhao X, Yang L, Hu J. Down-regulation of mir-27a might inhibit proliferation and drug resistance of gastric cancer cells. J Exp Clin Cancer Res. 2011; 30: 55.

27. Feng SY, Dong CG, Wu WK, et al. Lentiviral expression of anti-micrornas targeting mir-27a inhibits proliferation and invasiveness of u87 glioma cells. Mol Med Rep. 2012; 6: 275-81.

28. Ge YF, Sun J, Jin CJ, et al. Antagomir-27a targets foxo3a in glioblastoma and suppresses u87 cell growth in vitro and in vivo. Asian Pac J Cancer Prev. 2013; 14: $963-8$.

29. Xu W, Liu M, Peng X, et al. Mir-24-3p and mir-27a-3p promote cell proliferation in glioma cells via cooperative regulation of mxi1. Int J Oncol. 2013; 42: 757-66

30. Swartling FJ. Myc proteins in brain tumor development and maintenance. Ups J Med Sci. 2012; 117: 122-31.

31. Acunzo M, Romano G, Palmieri D, et al. Cross-talk between met and egfr in non-small cell lung cancer involves mir-27a and sprouty2. Proc Natl Acad Sci U S A. 2013; 110: 8573-8.

32. Yang Y, Zang A, Jia Y, et al. Genistein inhibits a549 human lung cancer cell proliferation via mir-27a and met signaling. Oncol Lett. 2016; 12: 2189-93.

33. Venkatesh T, Nagashri MN, Swamy SS, et al. Primary microcephaly gene mcph1 shows signatures of tumor suppressors and is regulated by mir-27a in oral squamous cell carcinoma. PLoS One. 2013; 8: e54643.

34. Sun B, Li J, Shao D, et al. Adipose tissue-secreted mir-27a promotes liver cancer by targeting foxo1 in obese individuals. Onco Targets Ther. 2015; 8: $735-44$

35. Li S, Li J, Fei BY, et al. Mir-27a promotes hepatocellular carcinoma cell proliferation through suppression of its target gene peroxisome proliferator-activated receptor gamma. Chin Med J (Engl). 2015; 128: 941-7.

36. Gao Y, Li BD, Liu YG. Effect of mir27a on proliferation and invasion in colonic cancer cells. Asian Pac J Cancer Prev. 2013; 14: 4675-8.

37. Chintharlapalli S, Papineni S, Abdelrahim M, et al. Oncogenic microrna-27a is a target for anticancer agent methyl 2-cyano-3,11-dioxo-18beta-olean-1,12-dien-30-oate in colon cancer cells. Int J Cancer. 2009; 125: 1965-74.

38. Fletcher CE, Dart DA, Sita-Lumsden A, et al. Androgen-regulated processing of the oncomir mir-27a, which targets prohibitin in prostate cancer. Hum Mol Genet. 2012; 21: 3112-27.

39. Peng $\mathrm{H}$, Wang $\mathrm{X}$, Zhang $\mathrm{P}$, et al. Mir-27a promotes cell proliferation and metastasis in renal cell carcinoma. Int J Clin Exp Pathol. 2015; 8: 2259-66.

40. Wu XZ, Wang KP, Song HJ, et al. Mir-27a-3p promotes esophageal cancer cell proliferation via f-box and wd repeat domain-containing 7 (fbxw7) suppression. Int J Clin Exp Med. 2015; 8: 15556-62.

41. Zeng G, Xun W, Wei K, et al. Microrna-27a-3p regulates epithelial to mesenchymal transition via targeting yap1 in oral squamous cell carcinoma cells. Oncol Rep. 2016; 36: 1475-82.

42. Lin SC, Kao CY, Lee HJ, et al. Dysregulation of mirnas-coup-tfii-foxm1-cenpf axis contributes to the metastasis of prostate cancer. Nat Commun. 2016; 7: 11418.

43. Salah Z, Arafeh R, Maximov V, et al. Mir-27a and mir-27a* contribute to metastatic properties of osteosarcoma cells. Oncotarget. 2015; 6: 4920-35.

44. Urbich C, Kaluza D, Fromel T, et al. Microrna-27a/b controls endothelial cell repulsion and angiogenesis by targeting semaphorin 6a. Blood. 2012; 119: $1607-16$

45. Lo TL, Yusoff $\mathrm{P}$, Fong CW, et al. The ras/mitogen-activated protein kinase pathway inhibitor and likely tumor suppressor proteins, sprouty 1 and sprouty 2 are deregulated in breast cancer. Cancer Res. 2004; 64: 6127-36.

46. Fong CW, Chua MS, Mckie AB, et al. Sprouty 2, an inhibitor of mitogen-activated protein kinase signaling, is down-regulated in hepatocellular carcinoma. Cancer Res. 2006; 66: 2048-58.

47. Qian P, Zuo Z, Wu Z, et al. Pivotal role of reduced let-7g expression in breast cancer invasion and metastasis. Cancer Res. 2011; 71: 6463-74.

48. Jiang X, Bailly MA, Panetti TS, et al. Formation of tissue factor-factor viia-factor xa complex promotes cellular signaling and migration of human breast cancer cells. J Thromb Haemost. 2004; 2: 93-101.

49. Wang YL, Gong WG, Yuan QL. Effects of mir-27a upregulation on thyroid cancer cells migration, invasion, and angiogenesis. Genet Mol Res. 2016; 15:gmr15049070. 
50. Tang $\mathrm{W}, \mathrm{Yu}$ F, Yao $\mathrm{H}$, et al. Mir-27a regulates endothelial differentiation of breast cancer stem like cells. Oncogene. 2014; 33: 2629-38.

51. Li X, Mertens-Talcott SU, Zhang S, et al. Microrna-27a indirectly regulates estrogen receptor \{alpha\} expression and hormone responsiveness in mcf-7 breast cancer cells. Endocrinology. 2010; 151: 2462-73.

52. Dhanabal M, Wu F, Alvarez E, et al. Recombinant semaphorin 6a-1 ectodomain inhibits in vivo growth factor and tumor cell line-induced angiogenesis. Cancer Biol Ther. 2005; 4: 659-68.

53. Varshavsky A, Kessler O, Abramovitch S, et al. Semaphorin-3b is an angiogenesis inhibitor that is inactivated by furin-like pro-protein convertases. Cancer Res. 2008; 68: 6922-31.

54. Zhou Q, Gallagher R, Ufret-Vincenty R, et al. Regulation of angiogenesis and choroidal neovascularization by members of microrna-23 27 24 clusters. Proc Natl Acad Sci U S A. 2011; 108: 8287-92.

55. Young JA, Ting KK, Li J, et al. Regulation of vascular leak and recovery from ischemic injury by general and ve-cadherin-restricted mirna antagonists of mir-27. Blood. 2013; 122: 2911-9.

56. Zhao N, Sun H, Sun B, et al. Mir-27a-3p suppresses tumor metastasis and vm by down-regulating ve-cadherin expression and inhibiting emt: An essential role for twist-1 in hcc. Sci Rep. 2016; 6: 23091.

57. Mertens-Talcott SU, Chintharlapalli S, Li X, et al. The oncogenic microrna-27a targets genes that regulate specificity protein transcription factors and the g2-m checkpoint in mda-mb-231 breast cancer cells. Cancer Res. 2007; 67: 11001-11.

58. Lai $Y$, Zhang $X$, Zhang Z, et al. The microrna-27a: Zbtb10-specificity protein pathway is involved in follicle stimulating hormone-induced vegf, cox2 and survivin expression in ovarian epithelial cancer cells. Int J Oncol. 2013; 42: 776-84.

59. Huang $Y$, Hua $K$, Zhou X, et al. Activation of the pi3k/akt pathway mediates fsh-stimulated vegf expression in ovarian serous cystadenocarcinoma. Cell Res. 2008; 18: 780-91.

60. Birchmeier C, Gherardi E. Developmental roles of hgf/sf and its receptor, the c-met tyrosine kinase. Trends Cell Biol. 1998; 8: 404-10.

61. Scagliotti GV, Selvaggi G, Novello S, et al. The biology of epidermal growth factor receptor in lung cancer. Clin Cancer Res. 2004; 10: 4227s-32s.

62. Fujino S, Enokibori T, Tezuka N, et al. A comparison of epidermal growth factor receptor levels and other prognostic parameters in non-small cell lung cancer. Eur J Cancer. 1996; 32A: 2070-4.

63. Maulik G, Kijima T, Salgia R. Role of receptor tyrosine kinases in lung cancer. Methods Mol Med. 2003; 74: 113-25.

64. Wong ES, Fong CW, Lim J, et al. Sprouty2 attenuates epidermal growth factor receptor ubiquitylation and endocytosis, and consequently enhances ras/erk signalling. EMBO J. 2002; 21: 4796-808

65. Holgren C, Dougherty U, Edwin F, et al. Sprouty-2 controls c-met expression and metastatic potential of colon cancer cells: Sprouty/c-met upregulation in human colonic adenocarcinomas. Oncogene. 2010; 29: 5241-53.

66. Fong CW, Leong HF, Wong ES, et al. Tyrosine phosphorylation of sprouty2 enhances its interaction with c-cbl and is crucial for its function. J Biol Chem. 2003; 278: 33456-64.

67. Toden S, Okugawa Y, Buhrmann C, et al. Novel evidence for curcumin and boswellic acid-induced chemoprevention through regulation of mir-34a and mir-27a in colorectal cancer. Cancer Prev Res (Phila). 2015; 8: 431-43.

68. Feng DD, Zhang H, Zhang P, et al. Down-regulated mir-331-5p and mir-27a are associated with chemotherapy resistance and relapse in leukaemia. J Cell Mol Med. 2011; 15: 2164-75.

69. Zhu $\mathrm{H}, \mathrm{Wu} \mathrm{H}$, Liu X, et al. Role of microrna mir-27a and mir-451 in the regulation of mdr1/p-glycoprotein expression in human cancer cells. Biochem Pharmacol. 2008; 76: 582-8.

70. Zhou S, Huang $\mathrm{Q}$, Zheng S, et al. Mir-27a regulates the sensitivity of breast cancer cells to cisplatin treatment via bak-smac/diablo-xiap axis. Tumour Biol. 2016; 37: 6837-45.

71. Zhao Q, Li Y, Tan BB, et al. Hif-1alpha induces multidrug resistance in gastric cancer cells by inducing mir-27a. PLoS One. 2015; 10: e0132746.

72. Drayton RM, Dudziec E, Peter S, et al. Reduced expression of mirna-27a modulates cisplatin resistance in bladder cancer by targeting the cystine/glutamate exchanger slc7a11. Clin Cancer Res. 2014; 20: 1990-2000.

73. Tanaka K, Miyata H, Sugimura K, et al. Mir-27 is associated with chemoresistance in esophageal cancer through transformation of normal fibroblasts to cancer-associated fibroblasts. Carcinogenesis. 2015; 36: 894-903.

74. Heinlein CA, Chang C. Androgen receptor in prostate cancer. Endocr Rev. 2004; 25: 276-308.

75. Wan X, Huang W, Yang S, et al. Androgen-induced mir-27a acted as a tumor suppressor by targeting map2k4 and mediated prostate cancer progression. The International Journal of Biochemistry \& Cell Biology. 2016; 79: 249-60.

76. Kourea HP, Zolota V, Scopa CD. Targeted pathways in breast cancer: Molecular and protein markers guiding therapeutic decisions. Curr Mol Pharmacol. 2014; 7: 4-21.

77. Dittrich A, Gautrey H, Browell D, et al. The her2 signaling network in breast cancer--like a spider in its web. J Mammary Gland Biol Neoplasia. 2014; 19: 253-70.

78. Lerner M, Lundgren J, Akhoondi S, et al. Mirna-27a controls fbw7/hcdc4-dependent cyclin e degradation and cell cycle progression. Cell Cycle. 2011; 10: 2172-83.

79. Spruck C. Mir-27a regulation of $\operatorname{scf}(\mathrm{fbw} 7)$ in cell division control and cancer. Cell Cycle. 2011; 10: 3232-3.
80. Zhang $\mathrm{S}$, Ma $\mathrm{C}$, Pang $\mathrm{H}$, et al. Arsenic trioxide suppresses cell growth and migration via inhibition of mir-27a in breast cancer cells. Biochem Biophys Res Commun. 2016; 469: 55-61.

81. Huang D, Wang H, Liu R, et al. Mirna27a is a biomarker for predicting chemosensitivity and prognosis in metastatic or recurrent gastric cancer. J Cell Biochem. 2014; 115: 549-56.

82. Tang $\mathrm{J}$, Zhao $\mathrm{H}$, Cai $\mathrm{H}$, et al. Diagnostic and prognostic potentials of microrna-27a in osteosarcoma. Biomed Pharmacother. 2015; 71: 222-6. 